\title{
Review Article \\ Huangqin-Tang and Ingredients in Modulating the Pathogenesis of Ulcerative Colitis
}

\author{
Chunyan Wang, ${ }^{1}$ Xudong Tang, ${ }^{2}$ and Li Zhang' \\ ${ }^{1}$ Institute of Digestive Diseases, China-Canada Center of Research for Digestive Diseases (ccCRDD), Longhua Hospital, \\ Shanghai University of Traditional Chinese Medicine, Shanghai 200032, China \\ ${ }^{2}$ Xiyuan Hospital of China Academy of Chinese Medical Sciences, Beijing 100091, China \\ Correspondence should be addressed to Li Zhang; zhangli.hl@163.com
}

Received 17 March 2017; Accepted 22 May 2017; Published 12 June 2017

Academic Editor: Luísa Mota da Silva

Copyright (C) 2017 Chunyan Wang et al. This is an open access article distributed under the Creative Commons Attribution License, which permits unrestricted use, distribution, and reproduction in any medium, provided the original work is properly cited.

Ulcerative colitis (UC) is the most common inflammatory bowel disease worldwide. Current therapies in UC cause limitations, and herb medicine provides an important choice for UC treatment. Huangqin-Tang (HQT) is a well-known classical traditional Chinese herbal formula and has been used in China for thousands of years. A large number of pharmacological studies demonstrated HQT and its ingredients to be effective in treating UC. Though the therapeutic effect has been evaluated, comprehensive up-todate reviews in this field are not yet available. Here we aim to review our current understanding of HQT and its ingredients in treating UC and how the agents modulate the main pathogenesis of the disease, including the intestinal environment, immune imbalance, inflammatory pathways, and oxidative stress. The summary on this issue may provide better understanding of HQT and its ingredients in treating UC and possibly help in promoting its clinical application.

\section{Introduction}

Ulcerative colitis (UC) is the most common form of inflammatory bowel disease, it is characterized by chronic inflammatory disorders of the colonic mucosa, which starts in the rectum and generally extends proximally in a continuous manner through part of or the entire colon [1]. The disease is bifurcated into remitting and relapsing courses [2] and may have significant impact on the quality of life and personal burden through reduction in the ability to work $[3,4]$. UC patients may require life-long treatment and have an increased risk of developing colorectal cancer $[5,6]$.

The global prevalence of UC is about 8 million, and the incidence and prevalence are increasing worldwide [7]. While the precise cause of UC is still unknown, it has been hypothesized that various factors such as geography, age, sex, genetic, environmental, gastrointestinal infection, and appendicectomy are responsible for the development of UC [1, 8-11]. The pathophysiology is related to epithelial barrier impairment, commensal microflora disorders, antigen recognition, dysregulation of immunological responses, leucocyte recruitment, and so forth. However, since the exact mechanisms of UC are still under investigation, treatment strategies are limited. The principle for UC treatment is divided into two categories according to the clinical activity and the extent of diseases, induction of remission, and maintenance of remission. The main agents consist of topical or oral mesalazine, oral or intravenous corticosteroids, immunosuppressive drugs, monoclonal antibodies of TNF$\alpha$, and colectomy [1]. However, the side effects and high cost limit the long-term application [12].

Recently, natural products have attracted lots of interest in preventing and treating UC. Traditional Chinese Medicines (TCM) and extracts have shown various beneficial treatment effects including bacteriostasis, anti-inflammation, and anticancer abilities [13]. Among them, Huangqin-Tang (HQT) is a well-known classical formula which derived from Shang Han Lun, consisting of four ingredients: the roots of Scutellaria baicalensis Georgi (scute), Glycyrrhiza uralensis Fisch (licorice), Paeonia lactiflora Pall. (peony), and the fruit of Ziziphus jujuba Mill (Chinese date). Using high-quality herbs picked by experienced herbalists and manufactured according to cGMP (current Good Manufacturing Practice), 


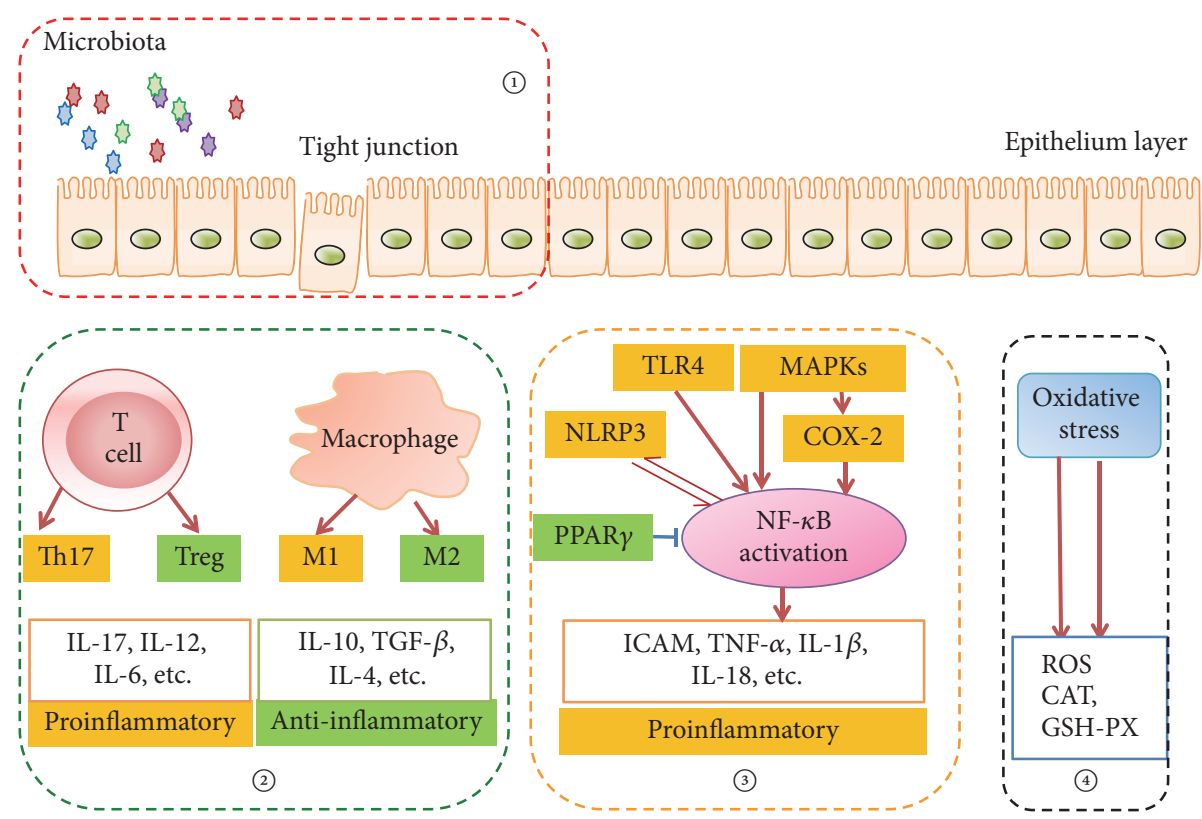

FIGURE 1: HQT regulates the main pathogenesis of UC. (1) HQT and its ingredients modulate intestinal microbiota and the integrity of epithelium; (2) HQT and its ingredients maintain the immune balance (Th17 versus Treg; M1 versus M2 type macrophages); (3) HQT and its ingredients target the inflammatory pathways, which could result in decrease of proinflammatory cytokines release; (4) HQT and its ingredients improve oxidative stress via enhancing antioxidants production. Note. $\uparrow$ indicates activated action and T indicates inhibited action.

HQT was extracted and named as PHY906 [14]. Using standardized chemical and biological fingerprints, the consistent preparations of PHY906 have been made and developed as an adjuvant for chemotherapy in various cancers.

Though HQT has been widely used in treating UC in China, the underlying mechanisms are still not clear. Pharmacokinetic studies on multiconstituents in HQT by the validated HPLC method showed that the main ingredients of HQT included baicalin, wogonoside, oroxylin-A-glucuronide, baicalein, wogonin, oroxylin-A, paeoniflorin, paeonimetabolin-I, liquiritin, liquiritigenin, glycyrrhizic acid, glycyrrhetinic acid, and visidulin I [15]. These constituents can have anti-inflammatory effect through different or similar mechanisms in UC treatment. Application of HQT or its ingredients in UC has been widely investigated; however, the systematic effect evaluation and mechanisms exploration are spotted, and we aim to summarize the current studies about the therapeutic effects or mechanisms of HQT or its main ingredients in UC (Figure 1), thus to better support its clinical use.

\section{HQT and Ingredients in Targeting Intestinal Environment on UC}

Imbalanced interactions with intestinal microbes lead to development of UC, and intestinal microbiota and epithelium provide environment for the pathogens.

There are approximately $10^{11} \sim 10^{14}$ enteric commensal microorganisms in the gut. Under normal conditions, these commensal bacteria maintain the balance and help in regulating crucial nutrient provision, immune response, and energy metabolism. However, commensal microorganisms can be noxious for intestinal inflammation under certain circumstances, and the diversity and amount of microbiota are reduced in patients with UC compared to that in healthy humans [14]. Though the specific bacteria related to the incidence of UC have not been found, lots of studies have showed that the change of intestinal environment was closely related to UC, and there was significantly different bacterial colonies between UC patients and healthy people [16-18]. PHY906, the extracted HQT, has been reported to alter the profile of major intestinal bacteria species, and treatment of mice with PHY906 could significantly decrease Bacteroides and E. rectale/C. coccoides and increase Clostridium leptum specially in the colon [19]. In addition, Paeonia lactiflora root, the main constituent of HQT, has been demonstrated to inhibit the growth of harmful intestinal bacteria in human [20].

The intestinal epithelial cells structurally constitute crypts and villi in the intestine, with a single columnar cell lining with a tight junction. The epithelial integrity is maintained by tight junctions between epithelial cells, and external pathogens easily pass through the injured or incomplete intestinal epithelium, which is known to be involved in the pathogenesis of UC. Several lines of evidence showed that the ingredients of HQT can protect the epithelium from pathogen introduction. Wogonin is a flavonoid isolated from Scutellaria baicalensis Georgi, the predominant ingredient of HQT. Pretreatment of Caco-2 cells with wogonin protects intestinal barrier function in lipopolysaccharide (LPS) stressed condition. Both $10 \mu \mathrm{M}$ and $50 \mu \mathrm{M}$ wogonin attenuate the LPS-induced transepithelial electrical resistance and transport of fluorescent markers and upregulates claudin1 and $\mathrm{ZO}-1$, the representative tight junction proteins in 
intestine [21]. Paeoniflorin, the main active ingredient of Paeonia lactiflora Pall., is confirmed to attenuate LPS-induced permeability in endothelial cells, and the ingredient (10, 30 , and $100 \mu \mathrm{M}$ ) could inhibit dextran extravasation and leukocyte migration in a concentration-dependent manner [22]. Furthermore, the ingredient glycyrrhetinic acid also exerted protective effects on LPS stressed intestinal epithelial cells injury and expression of the epithelial tight junction molecules [23].

\section{HQT and Ingredients in Regulating Immune Imbalance}

The gut possesses an abundant and highly active immune system that is tightly regulated to prevent overreaction of immune responses [24]. Studies have provided the evidences that the dysfunction of gut immune system, mainly involving the abnormal percentage and disturbed differentiation of immune cells, can result in the increase of proinflammatory cytokines, epithelial permeability, and subsequent intestinal mucosa damage [25-27]. Both Th17 and regulatory T (Treg) cells are originated from $\mathrm{CD} 4^{+} \mathrm{T}$ cells. Th17 cells, which secrete IL-17 and IL-22, promote inflammation process. On the contrary, Treg cells, which produces IL-10 and transforming growth factor- $\beta$ (TGF $\beta$ ), inhibit the inflammatory response $[28,29]$. As one important subtype of $\mathrm{T}$ cells, $\mathrm{CD} 4^{+} \mathrm{CD} 25^{+}$forkhead box $3^{+}{ }^{+}$Foxp $^{+}$) Treg cells inhibit autoimmunity and protect the tissue against injury and their development is controlled by Foxp3.

Growing evidences suggest that the imbalance between Th17 cells and Treg cells may contribute to the development of UC [30-32]. The ingredients of HQT are reported to regulate immune imbalance in a series studies. In trinitrobenzenesulfonic acid- (TNBS-) induced UC mice, baicalin could ameliorate the severity of the disease by downregulating the number of Th17 cells and the levels of Th17related cytokines (IL-17 and IL-6) and upregulating Treg cells and related TGF $\beta$, IL-10, and Foxp3 levels [33]. Similar effect of baicalin was also observed in cultured T cells; it proved that baicalin induced Foxp3 protein expression and inhibited $\mathrm{T}$ cells proliferation [34]. $\mathrm{CD} 4^{+} \mathrm{CD} 29^{+}$cells are helper $\mathrm{T}$ cells that can result in high activation of $\mathrm{B}$ cells and abnormal immune response. Previous study showed the percentage of $\mathrm{CD} 4^{+} \mathrm{CD} 29^{+} \mathrm{T}$ cells significantly increased in UC patients, indicating $\mathrm{CD} 4^{+} \mathrm{CD} 29^{+} \mathrm{T}$ cells might be an immunology index for monitoring UC $[35,36]$. Baicalin can reduce the percentages of $\mathrm{CD} 4^{+} \mathrm{CD} 29^{+} \mathrm{T}$ cells in cultured peripheral blood mononuclear cells from the UC patients, thus regulate immune balance, and relieve the UC-induced inflammation [37]. These studies inferred that baicalin may serve as a promising natural immunosuppressive compound for treating UC and related inflammatory diseases.

Macrophages play critical roles in both innate and adaptive immune responses and are classified into M1 and M2 phenotypes. M1 phenotype cells are stimulated by microbial products or other pathogens and produce many proinflammatory cytokines. In contrast, M2 type responses are the "resting" phenotype and are observed in healing-type circumstances without infections and generate anti-inflammatory cytokine. Macrophage M1 and M2 types execute opposite activities in tissues [38]. Once the M1/M2 balance is broken, immune-mediated inflammation occurs. Baicalin is reported to polarize macrophages to an M2 phenotype in murine peritoneum and ameliorate experimental inflammatory bowel disease in mice [39]. In mouse bone marrow precursors generated M1 and M2 cells, paeoniflorin inhibited LPS-induced M1 activity by reducing iNOS and NO production, whereas enhanced IL-4 provoked M2 function by upregulating Arg-1 production and activity [40], suggesting paeoniflorin can suppress M1 cells activity and enhance M2 cells function simultaneously. In addition, paeoniflorin is confirmed to significantly ameliorate the immune complex induced vascular damage, leucocyte infiltrates, and adhesion molecules expression [41]. The pharmaceutical effect of baicalin is reported to be associated with macrophage migration inhibitory factor downregulation, the quantity of macrophages, and the amount of $\mathrm{M}$ macrophage-related cytokines and macrophage inflammatory protein- $3 \alpha$ [42].

$B$ cells are important in the development of autoimmune disorders. Autoreactive B cells can present self-antigens to autoreactive $\mathrm{T}$ cells, produce autoantibodies and proinflammatory cytokines, and amplify inflammatory responses [25, $43,44]$. Inhibiting the abnormal activation of $\mathrm{B}$ cells is thought to be an effective strategy for UC treatment. It is reported that the $\mathrm{B}$ cell activation can be inhibited by paeoniflorin; in LPS-stimulated murine spleen B cells, paeoniflorin inhibits CD69/CD86 expression and B cell proliferation [45].

\section{HQT and Ingredients in Modulating Inflammatory Pathways}

Interactions between proinflammatory cytokines and their receptors lead to activation of intracellular signal transduction. And NF- $\kappa \mathrm{B}$ has been recognized as a critical target in inflammatory process. The extracellular stimulus, like Ag, LPS, growth factors and inflammatory cytokines, signals, and so forth, could trigger NF- $\kappa \mathrm{B}$ activation and induce inflammation in tissues. Therefore, inhibited NF- $\kappa \mathrm{B}$ activation is considered to be an effective strategy for UC treatment [46].

Toll-like receptor 4 (TLR4), a key receptor for commensal recognition in innate immunity, is overexpressed in inflamed colonocytes [47, 48], and TLR4-mediated signal transduction can evenly lead to NF- $\kappa \mathrm{B}$ activation [49]. In LPS stressed RAW264.7 cells, baicalin can block the TLR4/NF- $\kappa$ B pathway and inhibit IL-6 release and cell proliferation; in SpragueDawley rats, TNBS-induced UC status can be ameliorated by baicalin [50, 51]. In DSS-induced C57BL/6 mice, paeoniflorin could improve UC, and the beneficial effects are considered to be related to the downregulation of TLR4 expression and the blockage of NF- $\kappa$ B activation [52]. In addition, wogonin could attenuate the TLR4-mediated inflammatory response and maintain the single-layer membrane structure under LPS stressed $\mathrm{Caco} 2$ cells, and the positive effects might be achieved via TLR4-MyD88-TAK1-mediated NF- $\kappa$ B pathway [21].

Mitogen-activated protein kinases (MAPKs) are conserved among all eukaryotes and participate in multiple 
cellular processes including cell growth, proliferation, differentiation, migration, inflammation, and survival. Numerous studies have described an increased expression of MAPKs in IBD patients [53]. There are several studies focusing on the therapeutic effects for UC by regulating MAPKs. Glycyrrhetinic acid had the inhibitory effects on IL-8 production in intestinal epithelial cells through blocking MAPKs phosphorylation, followed by I-KB $\alpha$ degradation and NF- $\kappa \mathrm{B}$ activation [54]. Similarly, isoLQ, a chalcone found in licorice, could attenuate the DSS-induced colitis via suppressing MAPKs phosphorylation and NF- $\kappa \mathrm{B}$ activation in inflamed colon tissue [55]. COX-2, one isoform of cyclooxygenase (COX), can be inducted by activating MAPK activity $[56,57]$. And previous research has shown that the level of COX2 increased in the intestinal tissues of UC, and COX-2 inhibitor could relieve intestinal inflammation in rats with UC [58-60]. PF2405, a standardized fraction of S. baicalensis, can significantly inhibit TNF- $\alpha$ induced COX-2 expression through JNK1/2 dephosphorylation and p38 MAPK in HT29 cells and reduced the expression of proinflammatory cytokines and COX-2 in TNBS-induced colitis in female C57BL/6 mice [61].

Intercellular adhesion molecule 1 (ICAM-1) and TNF$\alpha$ are the representative cytokines in inflammatory injuries. ICAM-1 induces the migration and infiltration of inflammatory cells into the lesion, whereas TNF- $\alpha$ can alter vascular and intestinal permeability [62]. It is reported that diammonium glycyrrhizinate could reduce TNF- $\alpha$ and ICAM- 1 by inhibiting the NF- $\kappa \mathrm{B}$ activation and improve intestinal inflammatory injury in a rat model [63]. NLRP3 inflammasome is a key component of inflammatory process and its dysregulation contributes to UC. The synthesis and accumulation of NLRP3 inflammasome can be activated by $N F-\kappa B$ and lead to the secretion of IL- $1 \beta$ and IL-18. It is reported that WG (the glucuronide metabolite of wogonin) could ameliorate DSS-induced colitis via inhibiting NF$\kappa \mathrm{B}$ and NLRP3 inflammasome activation [64]. In addition, there are studies that focus on the inflammatory cytokines. Paeoniflorin can play anti-inflammatory action by increasing the level of IL-17 and decreasing the level of IL-10 in recombinant human IL- $1 \beta$-stimulated human peripheral blood mononuclear cells in vitro [65]. The glycyrrhizic acid could significantly reduce TNF- $\alpha$ and IL- $1 \beta$ levels in TNBSinduced colitis rats, and in vitro experiments indicated that glycyrrhizic acid could inhibit IL-6 and elevate IL-10 production in LPS-activated macrophages and significantly inhibit lymphocytes proliferation [66]. And similar effects are obtained in DSS-induced inflamed mucosa in rats [67].

Peroxisome proliferator-activated receptor $\gamma(\operatorname{PPAR} \gamma)$, a member of the nuclear receptor family, has been recognized as an endogenous regulator of intestinal inflammation. It is reported that activating PPAR $\gamma$ can inhibit NF- $\kappa \mathrm{B}$ activation. Oroxyloside, one of the ingredients of HQT, has been reported to activate PPAR $\gamma$ and prevent DSS-induced colitis through inhibiting $\mathrm{NF}-\kappa \mathrm{B}$ pathway [68]. Moreover, thioredoxin system is implicated in the regulation of NF- $\kappa \mathrm{B}$ transactivation potential; there is one study that focuses on the inhibition of baicalin on the thioredoxin system and finds that baicalein can suppress mitogen induced thioredoxin activity in the unclear compartment of lymphocytes, thus limiting $\mathrm{NF}-\kappa \mathrm{B}$ dependent inflammatory responses [69]. In addition, it has been reported that estrogen can exert anti-inflammatory effects. Based on this background, one study shows that baicalein has estrogen-like activity and inhibits LPS-induced inflammatory cytokine production via regulating $\mathrm{NF}-\kappa \mathrm{B}$ pathway, suggesting the potential efficacy in preventing inflammation related diseases [70]. Scutellariae Radix extract was effective in treating acute DSS-induced UC with improving macroscopic and histological damage scores and enhancing recovery of normal colonic secretory function [71].

\section{HQT Ingredients in Inhibiting Oxidative Stress}

The available evidence suggests that oxidative stress may be involved in the pathogenesis of UC $[72,73]$. The reactive oxygen species (ROS) are produced in excess by the inflamed mucosa and overwhelm the endogenous defenses in inflammatory intestinal diseases [74]. Among the masses of related products of oxidative stress, catalase (CAT) and phospholipid hydroperoxide glutathione peroxidase (GSH$\mathrm{Px}$ ) can reduce the intestinal damage by strengthening the oxidation resistance or reducing lipid hydroperoxides to their corresponding alcohols $[75,76]$. One study showed that the possible mechanisms of baicalin in protecting UC were associated with the inhibition of oxidative stress; baicalin could increase the activities of CAT and GSH-Px in LPSstimulated RAW264.7 cells and TNBS-induced UC rats [77].

\section{Conclusions and Perspectives}

In summary, available reports suggested that the mechanisms under the efficacy of HQT or its components on UC are related to intestinal environment improvement, immune modulation, and regulation of inflammatory pathways or cytokines (Figure 1). Since most data are interpreted from animal studies or in vitro experiments, the effects and mechanisms of HQT in UC patients remain to be explored or verified. In addition, most of the current studies are observational studies and the targets of the components remain unclear.

Though, along with the upgrade of the Chinese drugs ingredient detection tools, the compounds of Chinese herbal prescription have made some progress, its standardization is still the important obstacle for traditional Chinese medicine. And these studies are influenced by the quality, origin, and different processing method of single herb. Future studies should focus on the standardization of the compounds and the best compatibility in accordance with the disease database and traditional Chinese medicine database in order to achieve the best effect. In addition, different prescriptions for the acute phase or remission phase of UC patients should also be considered.

\section{Conflicts of Interest}

The authors declared no conflicts of interest. 


\section{Acknowledgments}

This work was supported by the Shanghai Rising-Star Program (no. 17QA1404000).

\section{References}

[1] I. Ordás, L. Eckmann, M. Talamini, D. C. Baumgart, and W. J. Sandborn, "Ulcerative colitis," The Lancet, vol. 380, no. 9853, pp. 1606-1619, 2012.

[2] Y. Yokoyama, K. Matsuoka, T. Kobayashi et al., "A largescale, prospective, observational study of leukocytapheresis for ulcerative colitis: Treatment outcomes of 847 patients in clinical practice," Journal of Crohn's and Colitis, vol. 8, no. 9, pp. 981-991, 2014.

[3] P. Kawalec, "Indirect costs of inflammatory bowel diseases: Crohn's disease and ulcerative colitis. A systematic review," Archives of Medical Science, vol. 12, no. 2, pp. 295-302, 2016.

[4] J. Sammut, J. Scerri, and R. B. Xuereb, "The lived experience of adults with ulcerative colitis," Journal of Clinical Nursing, vol. 24, no. 17-18, pp. 2659-2667, 2015.

[5] G. Rogler, "Chronic ulcerative colitis and colorectal cancer," Cancer Letters, vol. 345, no. 2, pp. 235-241, 2014.

[6] J. Gordillo, E. Cabré, E. Garcia-Planella et al., "Thiopurine Therapy Reduces the Incidence of Colorectal Neoplasia in Patients with Ulcerative Colitis. Data from the ENEIDA Registry," Journal of Crohn's \& colitis, vol. 9, no. 12, pp. 1063-1070, 2015.

[7] F. Carbonnel, J. F. Colombel, and J. Filippi, "Methotrexate is not superior to placebo for inducing steroid-free remission, but induces steroid-free clinical remission in a larger proportion of patients with ulcerative colitis," Gastroenterology, vol. 150, no. 2, pp. 380-388.e4, 2016.

[8] H. Khalili, E. S. Huang, A. N. Ananthakrishnan et al., "Geographical variation and incidence of inflammatory bowel disease among US women," Gut, vol. 61, no. 12, pp. 1686-1692, 2012.

[9] S. M. Quezada and R. K. Cross, "Association of age at diagnosis and ulcerative colitis phenotype," Digestive Diseases and Sciences, vol. 57, no. 9, pp. 2402-2407, 2012.

[10] H.-S. Lee, S. H. Park, S.-K. Yang et al., "Appendectomy and the clinical course of ulcerative colitis: A retrospective cohort study and a nested case-control study from Korea," Journal of Gastroenterology and Hepatology (Australia), vol. 30, no. 3, pp. 470-477, 2015.

[11] R. E. Childers, S. Eluri, C. Vazquez, R. M. Weise, T. M. Bayless, and S. Hutfless, "Family history of inflammatory bowel disease among patients with ulcerative colitis: A systematic review and meta-analysis," Journal of Crohn's and Colitis, vol. 8, no. 11, pp. 1480-1497, 2014.

[12] V. Ung, N. X. Thanh, K. Wong et al., "Real-life treatment paradigms show infliximab is cost-effective for management of ulcerative colitis," Clinical Gastroenterology and Hepatology, vol. 12, no. 11, pp. 1871-1878, 2014.

[13] G. Chen, Y. Yang, M. Liu et al., "Banxia xiexin decoction protects against dextran sulfate sodium-induced chronic ulcerative colitis in mice," Journal of Ethnopharmacology, vol. 166, pp. 149156, 2015.

[14] R. Tilton, A. A. Paiva, J.-Q. Guan et al., "A comprehensive platform for quality control of botanical drugs (PhytomicsQC): a case study of Huangqin Tang (HQT) and PHY906," Chinese Medicine, vol. 5, article 30, 2010.
[15] F. Zuo et al., "Pharmacokinetic study on the multi-constituents of Huangqin-Tang decoction in rats," Biological and Pharmaceutical Bulletin, vol. 26, no. 7, pp. 911-919, 2003.

[16] T. Yukawa, T. Ohkusa, T. Shibuya et al., "Nested culture method improves detection of fusobacterium from stool in patients with ulcerative colitis," Japanese Journal of Infectious Diseases, vol. 66, no. 2, pp. 109-114, 2013.

[17] T. Nomura, T. Ohkusa, I. Okayasu et al., "Mucosa-associated bacteria in ulcerative colitis before and after antibiotic combination therapy," Alimentary Pharmacology and Therapeutics, vol. 21, no. 8, pp. 1017-1027, 2005.

[18] T. Ohkusa, N. Sato, T. Ogihara, K. Morita, M. Ogawa, and I. Okayasu, "Fusobacterium varium localized in the colonic mucosa of patients with ulcerative colitis stimulates speciesspecific antibody," Journal of Gastroenterology and Hepatology (Australia), vol. 17, no. 8, pp. 849-853, 2002.

[19] W. Lam et al., "The number of intestinal bacteria is not critical for the enhancement of antitumor activity and reduction of intestinal toxicity of irinotecan by the Chinese herbal medicine PHY906 (KD018)," BMC Complementary and Alternative Medicine, vol. 14, 490 pages, 2014.

[20] L. T. M. Ngan, J.-K. Moon, J.-H. Kim, T. Shibamoto, and Y.-J. Ahn, "Growth-inhibiting effects of Paeonia lactiflora root steam distillate constituents and structurally related compounds on human intestinal bacteria," World Journal of Microbiology and Biotechnology, vol. 28, no. 4, pp. 1575-1583, 2012.

[21] W. Wang, T. Xia, and X. Yu, "Wogonin suppresses inflammatory response and maintains intestinal barrier function via TLR4MyD88-TAK1-mediated NF- $\kappa$ B pathway in vitro," Inflammation Research, vol. 64, no. 6, pp. 423-431, 2015.

[22] H. Xu, J. Song, X. Gao et al., "Paeoniflorin attenuates lipopolysaccharide-induced permeability of endothelial cells: Involvements of F-Actin expression and phosphorylations of PI3K/AKT and PKC," Inflammation, vol. 36, no. 1, pp. 216-225, 2013.

[23] L.-N. Hu, X.-Y. Fang, H.-L. Liu et al., "Protective effects of $18 \beta$-glycyrrhetinic acid on LPS-induced injury in intestinal epithelial cells," Chinese Journal of Natural Medicines, vol. 11, no. 1, pp. 24-29, 2013.

[24] T. T. MacDonald and G. Monteleone, "Immunity, inflammation, and allergy in the gut," Science, vol. 307, no. 5717, pp. 1920-1925, 2005.

[25] E. G. W. Schmidt, H. L. Larsen, N. N. Kristensen, S. S. Poulsen, M. H. Claesson, and A. E. Pedersen, "B cells exposed to enterobacterial components suppress development of experimental colitis," Inflammatory Bowel Diseases, vol. 18, no. 2, pp. 284-293, 2012.

[26] J. C. Hoving, F. Kirstein, N. E. Nieuwenhuizen et al., "B cells that produce immunoglobulin e mediate colitis in BALB/c mice," Gastroenterology, vol. 142, no. 1, pp. 96-108, 2012.

[27] D. Haribhai, J. Ziegelbauer, S. Jia et al., "Alternatively activated macrophages boost induced regulatory $\mathrm{T}$ and Th17 cell responses during immunotherapy for colitis," Journal of Immunology, vol. 196, no. 8, pp. 3305-3317, 2016.

[28] W. Jiang, J. Su, X. Zhang et al., "Elevated levels of Th17 cells and Th17-related cytokines are associated with disease activity in patients with inflammatory bowel disease," Inflammation Research, vol. 63, no. 11, pp. 943-950, 2014.

[29] E. Owaga, R.-H. Hsieh, B. Mugendi, S. Masuku, C.-K. Shih, and J.-S. Chang, "Th17 cells as potential probiotic therapeutic targets in inflammatory bowel diseases," International Journal of Molecular Sciences, vol. 16, no. 9, pp. 20841-20858, 2015. 
[30] S. Lim, S. Lee, J. Jeong, H. S. Choi, H. B. Chang, and D. Kim, "DW2007 ameliorates colitis and rheumatoid arthritis in mice by correcting Th17/Treg imbalance and inhibiting NF- $\kappa \mathrm{B}$ activation," Biomolecules \& Therapeutics, vol. 24, no. 6, pp. 638649, 2016.

[31] Y. Gong, Y. Lin, N. Zhao et al., "The Th17/Treg Immune Imbalance in Ulcerative Colitis Disease in a Chinese Han Population," Mediators of Inflammation, vol. 2016, Article ID 7089137, 2016.

[32] J. Yao, C. Wei, and J. Y. Wang, "Effect of resveratrol on Treg/Th17 signaling and ulcerative colitis treatment in mice," World Journal of Gastroenterology, vol. 21, no. 21, pp. 6572-6581, 2015.

[33] Y. Zou, S.-X. Dai, H.-G. Chi et al., "Baicalin attenuates TNBSinduced colitis in rats by modulating the Th17/Treg paradigm," Archives of Pharmacal Research, vol. 38, no. 10, pp. 1873-1887, 2015.

[34] J. Yang, X. Yang, and M. Li, "Baicalin, a natural compound, promotes regulatory T cell differentiation," BMC Complementary and Alternative Medicine, vol. 12, article 64, 2012.

[35] Y. Yokoyama, K. Fukunaga, Y. Fukuda et al., "Demonstration of low-regulatory CD25High+CD4+ and high-pro-inflammatory CD28-CD4+ T-cell subsets in patients with ulcerative colitis: Modified by selective granulocyte and monocyte adsorption apheresis," Digestive Diseases and Sciences, vol. 52, no. 10, pp. 2725-2731, 2007.

[36] Y. Zhu et al., " $\mathrm{CD} 4^{+} \mathrm{CD} 29^{+} \mathrm{T}$ cells are blamed for the persistent inflammatory response in ulcerative colitis," International Journal of Clinical and Experimental Pathology, vol. 8, no. 3, pp. 2627-2637, 2015.

[37] F.-Y. Yu, S.-G. Huang, H.-Y. Zhang et al., "Effects of baicalin in CD4 + CD29 + T cell subsets of ulcerative colitis patients," World Journal of Gastroenterology, vol. 20, no. 41, pp. 1529915309, 2014.

[38] N. Wang, H. Liang, and K. Zen, "Molecular mechanisms that influence the macrophage M1-M2 polarization balance," Frontiers in Immunology, vol. 5, article 614, 2014.

[39] W. Zhu, Z. Jin, J. Yu et al., "Baicalin ameliorates experimental inflammatory bowel disease through polarization of macrophages to an M2 phenotype," International Immunopharmacology, vol. 35, pp. 119-126, 2016.

[40] T. Zhai, Y. Sun, H. Li et al., "Unique immunomodulatory effect of paeoniflorin on type i and II macrophages activities," Journal of Pharmacological Sciences, vol. 130, no. 3, pp. 143-150, 2016.

[41] T. Chen, Z.-P. Guo, L. Wang et al., "Paeoniflorin suppresses vascular damage and the expression of E-selectin and ICAM-1 in a mouse model of cutaneous Arthus reaction," Experimental Dermatology, vol. 22, no. 7, pp. 453-457, 2013.

[42] S.-X. Dai, Y. Zou, Y.-L. Feng, H.-B. Liu, and X.-B. Zheng, "Baicalin down-regulates the expression of macrophage migration inhibitory factor (MIF) effectively for rats with ulcerative colitis," Phytotherapy Research, vol. 26, no. 4, pp. 498-504, 2012.

[43] J. L. Reyes, A. Wang, M. R. Fernando et al., "Splenic B cells from hymenolepis diminuta-infected mice ameliorate colitis independent of T cells and via cooperation with macrophages," The Journal of Immunology, vol. 194, no. 1, pp. 364-378, 2015.

[44] L. Wang, A. Ray, X. Jiang et al., "T regulatory cells and B cells cooperate to form a regulatory loop that maintains gut homeostasis and suppresses dextran sulfate sodium-induced colitis," Mucosal Immunology, vol. 8, no. 6, pp. 1297-1312, 2015.
[45] J. Zhang, H. Li, R. Huo et al., "Paeoniflorin selectively inhibits LPS-provoked B-cell function," Journal of Pharmacological Sciences, vol. 128, no. 1, pp. 8-16, 2015.

[46] A. K. Pandurangan, S. Ismail, Z. Saadatdoust, and N. M. Esa, "Allicin alleviates dextran sodium sulfate- (DSS-) induced ulcerative colitis in BALB/c mice," Oxidative Medicine and Cellular Longevity, vol. 2015, Article ID 605208, 13 pages, 2015.

[47] Y. Tan, K.-F. Zou, W. Qian, S. Chen, and X.-H. Hou, "Expression and implication of toll-like receptors TLR2, TLR4 and TLR9 in colonic mucosa of patients with ulcerative colitis," Journal of Huazhong University of Science and Technology - Medical Science, vol. 34, no. 5, pp. 785-790, 2014.

[48] M. Fukata et al., "Constitutive activation of epithelial TLR4 augments inflammatory responses to mucosal injury and drives colitis-associated tumorigenesis," Inflammatory Bowel Diseases, vol. 17, no. 7, pp. 1464-1473, 2011.

[49] M. A. Khan, C. Ma, L. A. Knodler et al., "Toll-like receptor 4 contributes to colitis development but not to host defense during Citrobacter rodentium infection in mice," Infection and Immunity, vol. 74, no. 5, pp. 2522-2536, 2006.

[50] L. Cui, L. Feng, Z. H. Zhang, and X. B. Jia, "The antiinflammation effect of baicalin on experimental colitis through inhibiting TLR4/NF- $\kappa \mathrm{B}$ pathway activation," International Immunopharmacology, vol. 23, no. 1, pp. 294-303, 2014.

[51] J. Feng et al., "Baicalin down regulates the expression of TLR4 and NFkB-p65 in colon tissue in mice with colitis induced by dextran sulfate sodium," International Journal of Clinical and Experimental Medicine, vol. 7, no. 11, pp. 4063-4072, 2014.

[52] J. Zhang, W. Dou, E. Zhang et al., "Paeoniflorin abrogates DSS-induced colitis via a TLR4-dependent pathway," American Journal of Physiology - Gastrointestinal and Liver Physiology, vol. 306, no. 1, pp. G27-G36, 2014.

[53] M. Coskun, J. Olsen, J. B. Seidelin, and O. H. Nielsen, "MAP kinases in inflammatory bowel disease," Clinica Chimica Acta, vol. 412, no. 7-8, pp. 513-520, 2011.

[54] O. H. Kang et al., "Inhibition of interleukin-8 production in the human colonic epithelial cell line HT-29 by 18 beta-glycyrrhetinic acid," International Journal of Molecular Medicine, vol. 15, no. 6, pp. 981-985, 2005.

[55] Y. H. Choi, J.-K. Bae, H.-S. Chae et al., "Isoliquiritigenin ameliorates dextran sulfate sodium-induced colitis through the inhibition of MAPK pathway," International Immunopharmacology, vol. 31, pp. 223-232, 2016.

[56] C. C. Chen et al., "Tumor necrosis factor-alpha-induced cyclooxygenase-2 expression via sequential activation of ceramide-dependent mitogen-activated protein kinases, and IkappaB kinase 1/2 in human alveolar epithelial cells," Molecular Pharmacology, vol. 59, no. 3, pp. 493-500, 2001.

[57] K. Subbaramaiah, W. J. Chung, and A. J. Dannenberg, "Ceramide regulates the transcription of cyclooxygenase-2: Evidence for involvement of extracellular signal-regulated kinase/c-Jun N-terminal kinase and p38 mitogen-activated protein kinase pathways," Journal of Biological Chemistry, vol. 273, no. 49, pp. 32943-32949, 1998.

[58] C. Jobin, O. Morteau, D. S. Han, and R. B. Sartor, "Specific NF- $\kappa$ B blockade selectively inhibits tumour necrosis factor- $\alpha$ induced COX-2 but not constitutive COX-1 gene expression in HT-29 cells," Immunology, vol. 95, no. 4, pp. 537-543, 1998.

[59] I. I. Singer, D. W. Kawka, S. Schloemann, T. Tessner, T. Riehl, and W. F. Stenson, "Cyclooxygenase 2 is induced in colonic epithelial cells in inflammatory bowel disease," Gastroenterology, vol. 115, no. 2, pp. 297-306, 1998. 
[60] K. M. Sakthivel and C. Guruvayoorappan, "Amentoflavone inhibits iNOS, COX-2 expression and modulates cytokine profile, NF- $\kappa \mathrm{B}$ signal transduction pathways in rats with ulcerative colitis," International Immunopharmacology, vol. 17, no. 3, pp. 907-916, 2013.

[61] W.-Y. Jiang, G. S. Seo, Y.-C. Kim, D. H. Sohn, and S. H. Lee, "PF2405, standardized fraction of Scutellaria baicalensis, ameliorates colitis in vitro and in vivo," Archives of Pharmacal Research, vol. 38, no. 6, pp. 1127-1137, 2015.

[62] A. F. Hagel et al., "Plasma histamine and tumour necrosis factoralpha levels in Crohn's disease and ulcerative colitis at various stages of disease," Journal of physiology and pharmacology, vol. 66, no. 4, pp. 549-556, 2015.

[63] H. Yuan, W.-S. Ji, K.-X. Wu, J.-X. Jiao, L.-H. Sun, and Y.-T. Feng, "Anti-inflammatory effect of Diammonium Glycyrrhizinate in a rat model of ulcerative colitis," World Journal of Gastroenterology, vol. 12, no. 28, pp. 4578-4581, 2006.

[64] Y. Sun, Y. Zhao, J. Yao et al., "Wogonoside protects against dextran sulfate sodium-induced experimental colitis in mice by inhibiting NF- $\kappa \mathrm{B}$ and NLRP3 inflammasome activation," Biochemical Pharmacology, vol. 94, no. 2, pp. 142-154, 2015.

[65] X. Dai, L.-W. Wang, X.-Y. Jia et al., "Paeoniflorin regulates the function of human peripheral blood mononuclear cells stimulated by rhIL- $1 \beta$ by up-regulating Treg expression," Immunopharmacology and Immunotoxicology, vol. 37, no. 3, pp. 252-257, 2015.

[66] Y. Liu, J. Xiang, M. Liu, S. Wang, R. J. Lee, and H. Ding, "Protective effects of glycyrrhizic acid by rectal treatment on a TNBS-induced rat colitis model," Journal of Pharmacy and Pharmacology, vol. 63, no. 3, pp. 439-446, 2011.

[67] T. Kudo, S. Okamura, Y. Zhang, T. Masuo, and M. Mori, “Topical application of glycyrrhizin preparation ameliorates experimentally induced colitis in rats," World Journal of Gastroenterology, vol. 17, no. 17, pp. 2223-2228, 2011.

[68] X. Wang, Y. Sun, Y. Zhao et al., "Oroxyloside prevents dextran sulfate sodium-induced experimental colitis in mice by inhibiting NF- $\kappa$ B pathway through PPAR $\gamma$ activation," Biochemical Pharmacology, vol. 106, pp. 70-81, 2016.

[69] R. S. Patwardhan, D. Sharma, M. Thoh, R. Checker, and S. K. Sandur, "Baicalein exhibits anti-inflammatory effects via inhibition of NF- $\kappa$ B transactivation," Biochemical Pharmacology, vol. 108, pp. 75-89, 2016.

[70] G.-W. Fan, Y. Zhang, X. Jiang et al., "Anti-inflammatory activity of baicalein in LPS-stimulated RAW264.7 macrophages via estrogen receptor and NF- $\kappa \mathrm{B}$-dependent pathways," Inflammation, vol. 36, no. 6, pp. 1584-1591, 2013.

[71] H.-L. Chung, G. G.-L. Yue, K.-F. To, Y.-L. Su, Y. Huang, and W.-H. Ko, "Effect of Scutellariae Radix extract on experimental dextran-sulfate sodium-induced colitis in rats," World Journal of Gastroenterology, vol. 13, no. 42, pp. 5605-5611, 2007.

[72] A. Tuzun et al., "Oxidative stress and antioxidant capacity in patients with inflammatory bowel disease," Clinical Biochemistry, vol. 35, no. 7, pp. 569-572, 2002.

[73] A. Rezaie, R. D. Parker, and M. Abdollahi, "Oxidative stress and pathogenesis of inflammatory bowel disease: an epiphenomenon or the cause?" Digestive Diseases and Sciences, vol. 52, no. 9, pp. 2015-2021, 2007.

[74] A. D. Millar, D. S. Rampton, C. L. Chander et al., "Evaluating the antioxidant potential of new treatments for inflammatory bowel disease using a rat model of colitis," Gut, vol. 39, no. 3, pp. 407-415, 1996.
[75] S.-K. Ku, B.-I. Seo, and J.-H. Park, "Effect of Lonicerae Flos extracts on reflux esophagitis with antioxidant activity," World Journal of Gastroenterology, vol. 15, no. 38, pp. 4799-4805, 2009.

[76] P. A. Olsvik, T. Kristensen, R. Waagbø et al., "MRNA expression of antioxidant enzymes (SOD, CAT and GSH-Px) and lipid peroxidative stress in liver of Atlantic salmon (Salmo salar) exposed to hyperoxic water during smoltification," Comparative Biochemistry and Physiology - C Toxicology and Pharmacology, vol. 141, no. 3, pp. 314-323, 2005.

[77] J. Yao, X. Cao, R. Zhang et al., "Protective effect of baicalin against experimental colitis via suppression of oxidant stress and apoptosis," Pharmacognosy Magazine, vol. 12, no. 47, pp. 225-234, 2016. 


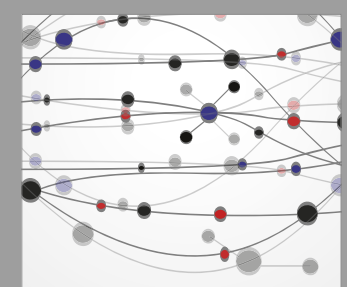

The Scientific World Journal
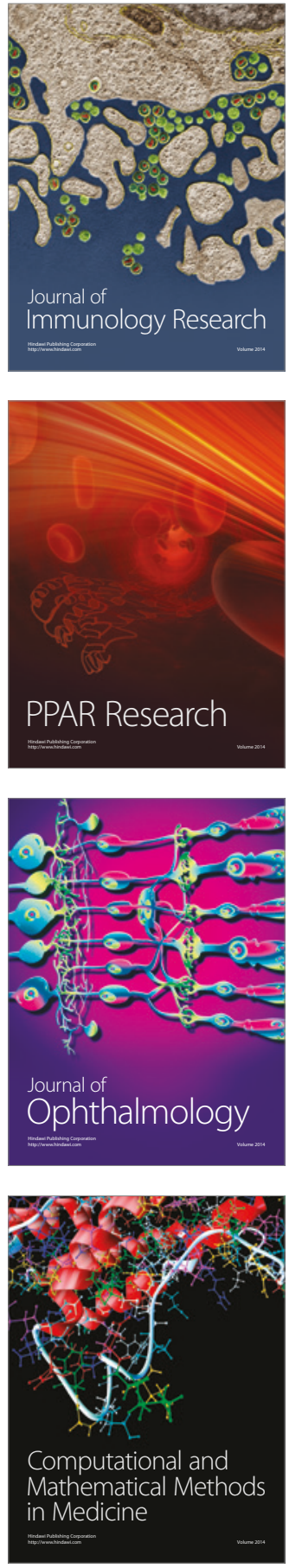

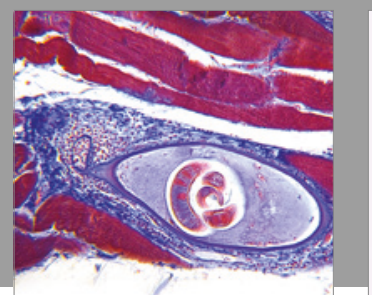

Gastroenterology Research and Practice
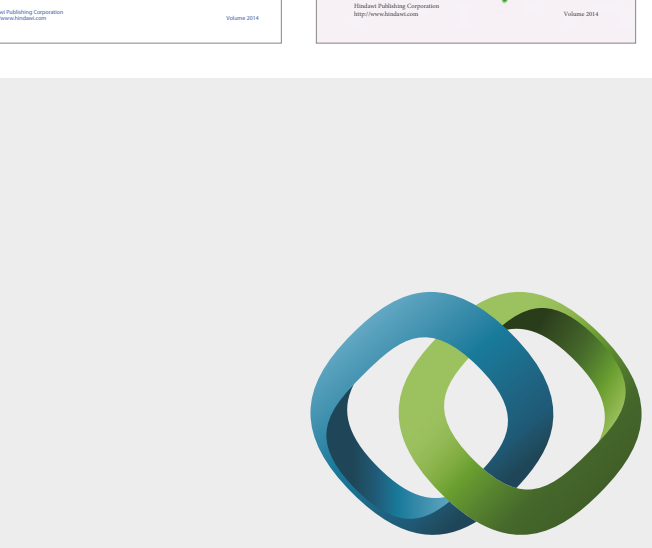

\section{Hindawi}

Submit your manuscripts at

https://www.hindawi.com
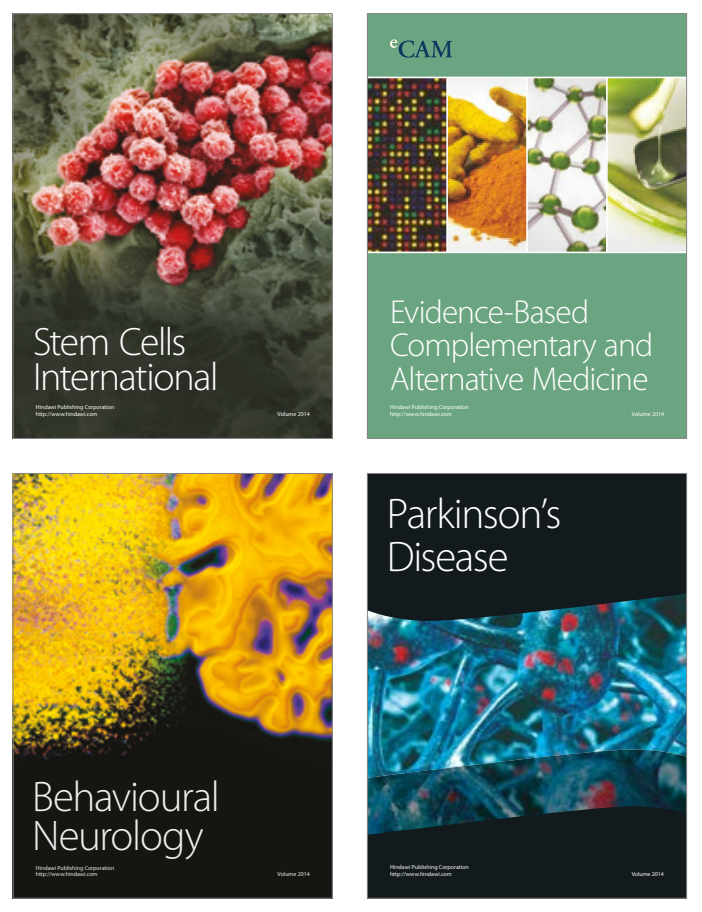
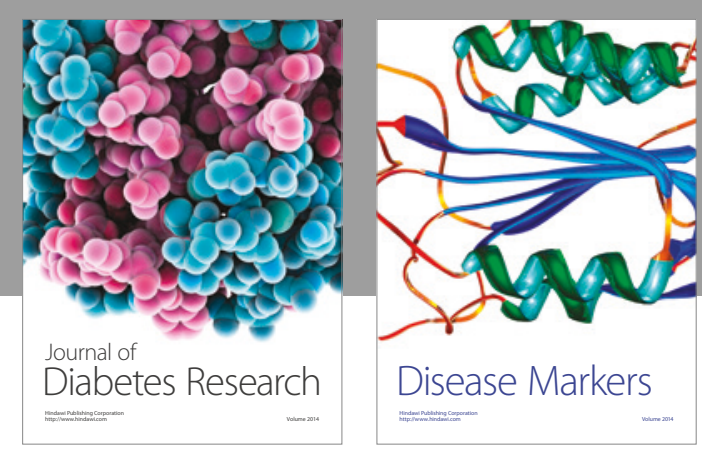

Disease Markers
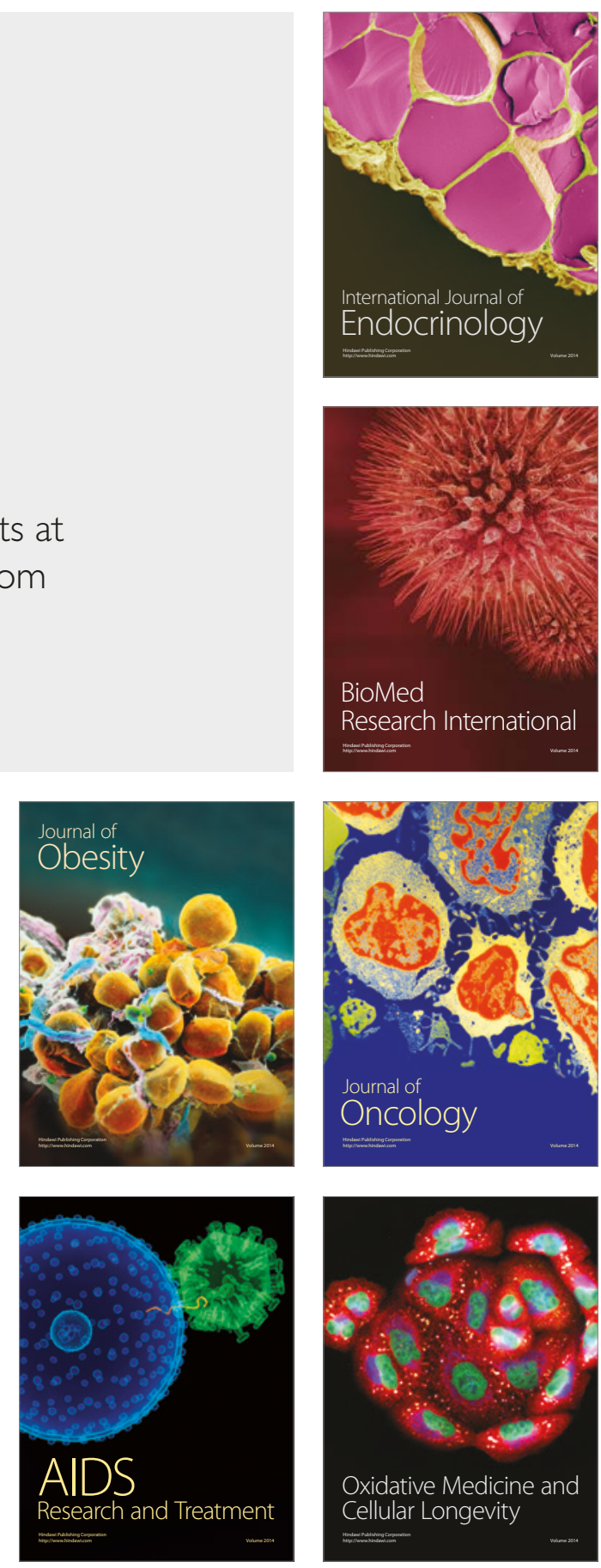